Results 400 surveys were returned from 402 women recruited (response rate $>99 \%$ ), age range 17-57 (mean 25.2) years.

$75 \%$ disagreed/strongly disagreed with the statement 'taking my own samples was difficult'. $72 \%$ agreed/strongly agreed that they felt confident taking their own swabs but $30.5 \%$ agreed/ strongly agreed they felt uncomfortable taking their own swabs; of these $53(43 \%)$ stated they had never had anal sex. $42 \%$ agreed/strongly agreed that they would prefer to take their own samples compared to $34 \%$ who agreed/strongly agreed they would prefer clinician-taken swabs. $66 \%$ agreed/strongly agreed they would be happy to take the samples themselves in a nonclinic setting.

Free comments covered themes of 'more confidence if had clinician samples taken before', 'concerns if self-swabbing would give correct results'. Nine commented specifically on discomfort, but none disagreed with the statement 'I would be happy to take my own swabs in a non-clinic environment'.

Conclusion Extra-genital sampling was highly acceptable to the majority of women, with high levels of confidence and low reports of discomfort. This has positive implications for the future of extra-genital testing in women, especially in non-clinic settings.

Disclosure of interest statement Dr Janet Wilson has received honoraria and travel and accommodation expenses from BD Diagnostics, and research grants in the form of diagnostic kits from Hologic/Gen-Probe.

\section{P13.12 HIV SENSITISATION HEALTH CARE WORKER TRAINING IN VANUATU}

K Coote, K Kishore, N Bates, P Prasad*.

\subsection{6/sextrans-2015-052270.510}

Introduction College of Medicine, Nursing and Health Sciences (CMNHS) in collaboration with The Albion Centre (Albion) conducted a 2-day HIV Sensitisation workshop in Port Vila, Vanuatu from $10^{\text {th }}-11^{\text {th }}$ March 2015. The HIV Sensitisation training is an activity of the Pacific Health Worker Support Project.

The HIV Sensitisation training aims to positively reshape health workers' perceptions about HIV and people from key populations (KP), including sex workers, men who have sex with men and PLHIV, by:

- Addressing shortfalls in knowledge and understanding about HIV;

- Demystifying/clarify personally-held attitudes and beliefs about HIV and PLHIV; and

- Learning about the perspectives and experiences of KP.

Methods This training was provided by two representatives from KP groups, who had previously completed training-of-trainers (ToT) workshops in Suva, Fiji.

Results Altogether 24 health workers, including representation from all six Vanuatu provinces attended the two-day workshop. The workshop was evaluated using two mechanisms: a Knowledge, Attitudes and Practices Survey (KAPS) administered to participants at pre and post training; and an anonymous course evaluation form, administered to participants at post training. Participants demonstrated improvement in all areas; however a paired-samples t-test analysis indicated that this improvement was significant only in the area of improved knowledge, across the group. Some of the key themes which emerged from the evaluations included that the training: raised their awareness of
$\mathrm{KP}$ and challenged their personal attitudes; heightened their awareness of how stigmatising language and behaviour can alienate $\mathrm{KP}$; and was needed through-out the healthcare workforce in Vanuatu.

Conclusion Overall the HIV Sensitisation Training workshop in Vanuatu was a success. The 2-day training raised their awareness of KP and challenged their personal attitudes; heightened their awareness of how stigmatising language and behaviour can alienate $\mathrm{KP}$; and was needed through-out the healthcare workforce in Vanuatu.

Disclosure of interest statement The Pacific Sexual Health and HIV Health Worker Support Project is funded by Australian aid's Regional HIV/AIDS Capacity Building Program (20122015). No pharmaceutical grants were received in the development of this study.

\section{P13.13 SEXUALLY TRANSMITTED INFECTION CARE IN TERMINATION OF PREGNANCY CLINICS IN THE NETHERLANDS}

${ }^{1} \mathrm{G}$ Van den Berg, ${ }^{2} \mathrm{C}$ Picavet, ${ }^{3} \mathrm{R}$ Hoopman, ${ }^{4} \mathrm{P}$ Lohr, ${ }^{1} \mathrm{E}$ Op de Coul ${ }^{*} .{ }^{1}$ Centre for Infectious Disease Control (RIVM), The Netherlands; ${ }^{2}$ Rutgers WPF, The Netherlands; ${ }^{3}$ Athena Institute for Research on Innovation and Communication in Health and Life Sciences, VU University, The Netherlands; ${ }^{4}$ British Pregnancy Advisory Service, UK

\subsection{6/sextrans-2015-052270.511}

Introduction Women attending termination of pregnancy (TOP) clinics are a risk group for STIs, and those with STIs are at increased risk of developing postabortal complications. We explored what STI care is provided in Dutch TOP clinics, and we compared these results with the United Kingdom (UK).

Methods A qualitative study including 14 semi-structured interviews with health care professionals (HCPs) in TOP clinics (Netherlands: 9, UK: 5). Interviews were recorded, transcribed, and analysed by thematic content analysis.

Results In the Netherlands, azithromycin prophylaxis is routinely prescribed after a surgical TOP, but not routinely after a medical TOP. STI tests are only offered by Dutch clinics if clients are considered at high STI risk based on the intake questionnaire. High costs of STI testing form the main barrier for clients not accepting STI testing, as costs are not covered by all health insurances. Alternatively, some clinics refer women to the Public Health Service (PHS), where STI testing is free for high-risk groups. This also involves barriers as not all women go for testing. HCPs in TOP clinics that collaborate with PHSs in the Sense program (sexual and reproductive health care) experience less barriers, as free STI testing is then offered on location of the TOP clinic. Sexual health counselling in TOP clinics is often limited to birth control conversations, also in the UK. The major difference between Dutch and UK TOP clinics is that UK clinics (who are not privately owned) offer free STI testing to all clients.

Conclusion HCPs in Dutch TOP clinics consider STI testing an important part of their service, but financial barriers prevent STI testing on location. In the UK, free STI care is broadly implemented in TOP clinics. Collaboration with PHSs (in Sense program) could improve STI testing and sexual health counselling in Dutch TOP clinics.

Disclosure of interest statement The study is funded by the RIVM. No pharmaceutical grants were received in the development of this study. 